\title{
An Energy Efficient Method for Tracking Mobile Ubiquitous Robots Using Wireless Sensor Network
}

\author{
Hyunsook Kim ${ }^{1}$, Jeongho Son ${ }^{1}$, Sukgyu Lee ${ }^{2}$, and Kijun Han ${ }^{1, *}$ \\ ${ }^{1}$ Department of Computer Engineering, \\ Kyungpook National University, 1370, Sangyuk-dong, Book-gu, \\ Daegu, Korea 702-010 \\ \{hskim, jhson\}@netopia.knu.ac.kr \\ ${ }^{2}$ Department of Electrical Engineering, \\ Yeungnam University, 214-1, Dae-dong, Gyongsan, Korea 712-749 \\ sglee@yu.ac.kr, kjhan@knu.ac.kr
}

\begin{abstract}
In general, it requires lots of complicated and expensive processing functions to find the exact location of a mobile target. For example, Ubiquitous Robotic Companion (URC) system should be equipped with powerful resources or be given an aid from the external servers to find its location for itself. Sensor network that consists of inexpensive low-power sensors can provide an efficient solution to find the exact location of such a mobile target at a low price. In such applications, if all sensor nodes have to always wake up to find location of the mobile robot, we have to pay a lot of waste of resources such as battery power and channel utilization. In this paper, we propose a cheap and energy efficient location tracking method of a mobile robot by minimizing the number of sensor nodes participating in the task of target tracking.
\end{abstract}

Keywords: Wireless sensor, target tracking, prediction, ubiquitous robot.

\section{Introduction}

Sensor networks consist of small and inexpensive low-power sensors, which facilitate monitoring and collecting information of sensor field. The sensors are used to monitor and control the physical environments and transmit the collected information to sink along optimized routing path. Wireless sensor network applications include such as battlefield surveillance, disaster and emergency response and traffic monitoring.

The paradigm shift of robotics is motivated by ubiquitous computing and sensor network where every device should be networked; computers should be accessible at anytime and at any place; and ubiquitous devices should be provide services suitable to the specific situation. Ubiquitous Robotic Companion (URC), a $3^{\text {rd }}$ generation of robotics, provides us with various services in a ubiquitous computing environment proposed as a concept that computer are embedded everywhere [1]. Since it is inherently based on ubiquitous environment with networked sensors and actuators, it can be considered as one of the most important emerging applications of sensor network.

\footnotetext{
* Correspondence author.
} 
Fig. 1 depicts the concept of URC with sensing, processing and acting abilities in wireless sensor network to overcome the technical constraints and producing costs by utilizing sensors and remoter computer server.

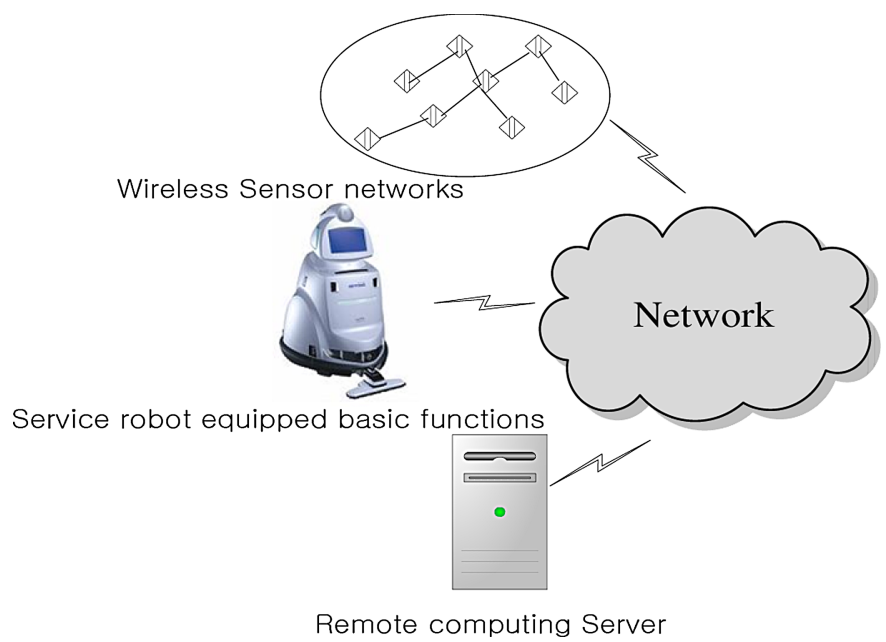

Fig. 1. The concept of the Ubiquitous Robotic Companion(URC)

By using network with external cheap sensors embedded in the environment replacing multiple robots equipped sensors, the context-awareness of the robot would be dramatically improved and lessen the burden of hardware cost [2]. In addition, remote computing server can be used as an external memory and processor of URC, which improves the robot intelligence and expands its applications and services.

URC system should be aware of its exact location at any time to perfectly carry out its mission. So, it has to be equipped with powerful resources or be given an aid from the external servers to find its location for itself since it requires lots of complicated and expensive processing functions to find its exact location. Sensor network can provide an efficient solution to find the exact location of such a mobile target at a low price. In such applications, if all sensor nodes have to always wake up to find location of the mobile robot, we have to pay a lot of waste of resources such as battery power and channel utilization.

Deciding the location of the mobile robot does not need all sensor nodes to work together. Instead, it is desirable that only several nodes surrounding the mobile robot should be responsible for observing the target to save the energy consumption and extend the network lifetime. In this paper, we propose a cheap and energy efficient location tracking method of a mobile robot by minimizing the number of sensor nodes participating in the task of target tracking. Our tracking method predicts the location of the mobile target in 2-dimensional wireless sensor network, based on linear estimation.

The rest of this paper is organized as follows. Section 2 summarizes the related works. The detailed of the proposed scheme is presented in section 3. Next, in section 4, we present some simulation results. Finally, section 5 concludes the paper. 


\section{Related Works}

\subsection{Localization}

It is fundamental to know the location of robots for performing their missions, so called localization. Sensor network may play an important role to enhance the resolution of location of URC by analyzing information on locations from sensors, for which some forms of communication between reference and the receiver are needed. Some typical examples of communication technologies in sensor network include RFbased, and acoustic based communication. In RF-based localization system, distance is estimated based on received signal strength. Since RF is sensitive to noise, Cricket uses both concurrent radio and ultrasonic sounds to enhance the resolution of distance estimation. Niculescu et. al proposed a technique based on angle-of-arrival and its enhanced version, range-free techniques to estimate position. A straightforward localization approach would make use of Global Positioning System (GPS). Existing research projects such as zebra-net uses a GPS based localization, where mobile sensors find out their location every three minutes. However, the positioning systems based on GPS alone face great problems in the so-called urban canyon environments, where GPS signals are often blocked by highrise buildings and there are not enough available satellite signals to estimate the positioning information of a fix. Bulusu et. al studied signal strength based and connectivity based techniques for localization in outdoor environments. Recently Kumar et. al proposed using dead reckoning-based location services for mobile ad hoc networks [3].

\subsection{Sensor Network in URC System}

When a mobile robot moves around far away from the sensing range of a certain node, the nods does not need to keep wake up for participating in tracking of the mobile robot. This raises the necessity for prediction of the moving path of the mobile robot to maintain the number of participating nodes in tracking as small as possible. Many tracking protocols in large-scale sensor networks have been proposed an energy efficient tracking scheme from various angles [4] [5] [6]. Krishnamurthy et al. [7] [8] proposed an energy efficient technique for using a sleep schedule where the nodes go to the sleep state when there is no need to take part in sensing. In [9], they explored a localized prediction approach to power efficient object tracking by putting unnecessary sensors in sleep mode. They proposed a convey tree for object tracking using data aggregation to reduce energy consumption. In [5], they attempt to solve the problem of energy savings based on the estimating the location of a mobile target. And they studied the frequency of tracking. We apply above sleep scheduling mechanisms to our study basically.

Actually, power conservation is one of the most critical issues in wireless sensor networks since the sensor nodes that are once deployed in the sensor field would be difficult to replace a battery. With rapid advances in sensor fabrications, recent sensors are designed to be power-aware, changing their condition (e.g., shut down sensing processor or radio) when they do not need to run the components to perform a given task in a sensor field. Most sensors can operate under the three different conditions: Active, Idle and Sleep. It is important to completely shut down the radio rather 
than put it in the idle mode when it does not sensing. Power management of sensor components is very important because energy consumption depends on their duties. For example, the amount of energy consumption at sensor node is shown in Fig.2.

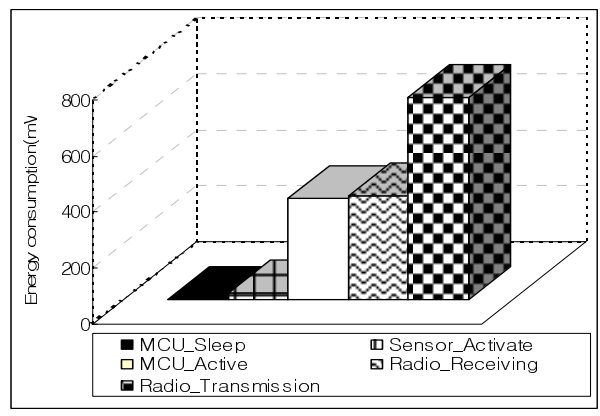

Fig. 2. Power consumption at a sensor node

So, if each node uses timely its energy to execute tasks, the network lifetime may be extended as a whole. Therefore, each sensor must minimize its battery power usage for desired longevity of network operation, which can be accomplished by properly managing sensor's operation.

\section{Finding Location of URC}

To save energy resources and thus extend the network lifetime, it is desirable that only several nodes surrounding the mobile robot join the task of observing the target.

For example, when the target passes through the ${ }^{t_{1}}$ point as shown in Fig. 3 , all nodes do not need to join the task for determining location and provide the results to the robot and to the external server.

Instead, it is more desirable that only several nodes around the mobile object join the task of collecting information of the robot and performing collaborative work among them. Other nodes located far from the target do not need to waste their powers to monitor the robot. If we can predict the next location of the mobile robot in advance, we can dynamically organize the group membership which should join the tracking mission. For example as shown in Fig. 3, if we predict future location of the mobile target accurately, the number of participating nodes can be minimized and thus the whole network lifetime can be extended.

As the mobile robot moves, the sensor nodes may migrate to the moving direction of the robot to keep on monitoring as shown in Fig. 3, where a thick line indicates the moving path of the mobile target and the blacked circles inside the dotted circle are tracking nodes at time ${ }^{t_{1}}$. Thus, sensor nodes need to control their states by themselves based on prediction of robot's movement. 


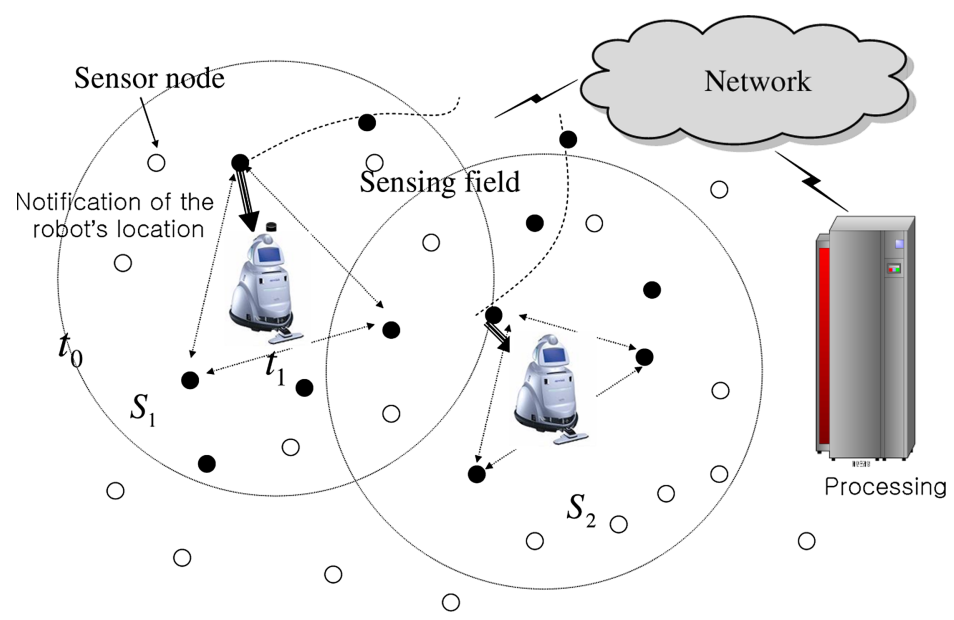

Fig. 3. Finding Location of URC in wireless sensor networks

We assume a sensor network where $\mathrm{N}$ sensors with the same communication and sensing range are distributed randomly in the environment that is being monitored. We also assume that each node knows its own location by using GPS or other location awareness techniques. And we utilize triangulation for localization of a mobile robot. Consequently, at least 3 sensors join the target detection and tracking with surveillance. Also each node keeps information about its neighbors such as location through the periodically message change. And each individual sensor node is equipped with appropriate sensory devices to be able to recognize the target as well as to estimate its distance based on the sensed data.

Further, we assume that we predict the location of the mobile targets every one second (or minute), and each sensor records the movement pattern of the mobile object. Basically, we use a moving average estimator to predict the future location of the mobile target based on the measurement of direction and the velocity of the mobile target.

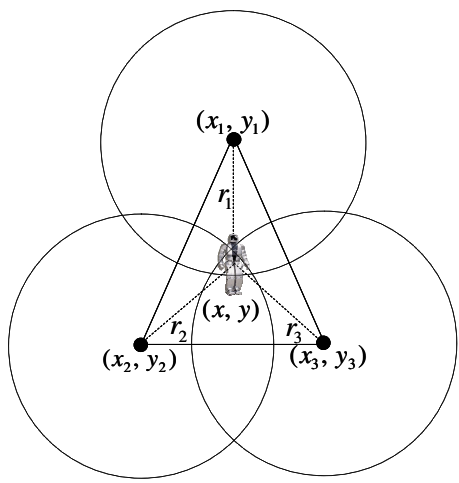

Fig. 4. Decision of location of a mobile robot using triangulation 
We assume that the sensor nodes are deployed randomly in a sensor field and each sensor senses the environment and communicates its readings to the server periodically; the server triangulates the location of the object using the readings [19].

Consider three sensors whose locations $\left(\left(x_{1}, y_{1}\right),\left(x_{2}, y_{2}\right),\left(x_{3}, y_{3}\right)\right)$ are known. We then have

$$
\left\{\begin{array}{l}
\left(x-x_{1}\right)^{2}+\left(y-y_{1}\right)^{2}=r_{1}^{2} \\
\left(x-x_{2}\right)^{2}+\left(y-y_{2}\right)^{2}=r_{2}{ }^{2} \\
\left(x-x_{3}\right)^{2}+\left(y-y_{3}\right)^{2}=r_{3}{ }^{2}
\end{array}\right.
$$

where $r$ is the Euclidean distance between the robot and a sensor node. This relation is the basic equation used in triangulation.

Discovery: When a sensor node around the mobile object detects the mobile robot, it becomes a 'master node' which acts as a header node temporarily.

Computation of location: A set of nodes which become aware of the appearance of the mobile robot compute the robot's current position by the triangulation which requires a collaborative work among at least three sensor nodes.

Prediction: The master node provides the location information to the mobile robot and the server computer along the optimized routing path. A master node predicts the future movement path of the mobile robot, and transmits information about the approaching location to its neighbor nodes. The prediction is carried out by two steps: an approximate prediction and a correction step that are explained in [18]. As the mobile target moves, each node hands off an initial estimate of the target location to the next node in turn. At that time, each node changes its duty cycle along the movement of the target [14].

Fig. 5. Tracking procedure of the mobile robot

Solving for $\mathrm{x}$ and $\mathrm{y}$, we get

$$
\begin{aligned}
& x=\frac{-b_{2} c_{1}+b_{1} c_{2}}{b_{2} a_{1}-b_{1} a_{2}} \\
& y=\frac{-a_{2} c_{1}+a_{1} c_{2}}{a_{2} b_{1}-a_{1} b_{2}}
\end{aligned}
$$


where

$$
\begin{gathered}
a_{1}=2\left(x_{2}-x_{1}\right), b_{1}=2\left(y_{2}-y_{1}\right), c_{1}=x_{1}{ }^{2}-x_{2}{ }^{2}+y_{1}{ }^{2}-y_{2}{ }^{2}-r_{1}{ }^{2}-r_{2}{ }^{2}, \\
a_{2}=2\left(x_{3}-x_{2}\right), b_{1}=2\left(y_{3}-y_{2}\right), c_{1}=x_{2}{ }^{2}-x_{3}{ }^{2}+y_{2}{ }^{2}-y_{3}{ }^{2}-r_{2}{ }^{2}-r_{3}{ }^{2}
\end{gathered}
$$

Tracking in our system is performed by the following procedure.

For energy saving, each node operates the state scheduling by itself. For example, detectable nodes within sensing range of a node ' $R$ ' in Fig. 6, which is near the target are activated, and they are participated in tracking including localization, monitoring, and prediction.

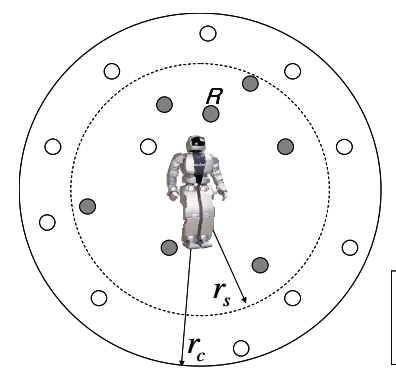

(a) Before moving

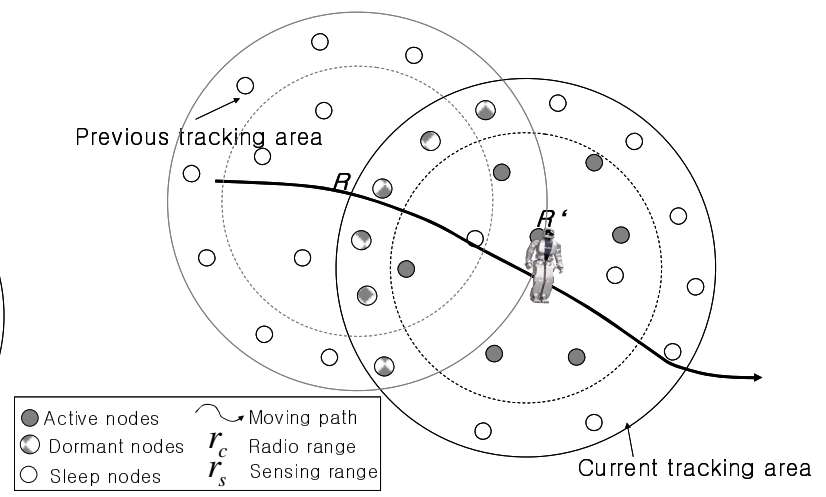

(b) After moving

Fig. 6. Change of state as the mobile target moves

So they consume amount of energy i.e., processor and sensor activating, transmitting and receiving. And the dormant node ' $R$ " can hear message about appearances of the mobile target in neighborhood, but cannot detect the target since the sensing range differs from its communication range. Obviously, all dormant nodes do not need to be activated. Energy consumed by the dormant nodes is small enough to be negligible comparing with that by the activated nodes. Some nodes those are located around the next position of the target wait for sensing. The others shut down the radio after hearing the message from the master node and they wake up from sleep when their duty cycle is over. When it wakes up, it first senses its region and hears message. If a sensor node cannot detect the target and does not receive any message for target appearance, it turns off its radio and goes to sleep for saving energy. And the rest of the sensor nodes run independently according to their duty cycles. Thus, the prediction of the mobile target in the tracking system reduces energy consumption in each node and extends network lifetime. 


\section{Simulation}

We evaluate the performance of our method through simulation results. We carry out experiments to measure missing rate and wasted energy. The network dimension for our experiments is [200, 200] and 500 nodes are randomly deployed within the region. And we assume that the sensing range of a sensor node is 20 and communication range is 35 . To model the movement behavior of the mobile target, we use the Random Way Point model (RWP) and Gauss-Markov mobility model. RWP is a simple mobility model based on random directions and speeds. The mobile object begins by staying in one location for a certain period of time. Once this time expires, the mobile object chooses a random destination in the field and a speed. And then travels toward the newly chosen destination at the selected speed. Upon arrival, the mobile object pauses for a specified time period before starting the process again. Gauss-Markov Mobility Model is a model that uses one tuning $(\alpha)$ parameter to vary the degree of randomness in the mobility pattern. Initially, each mobile node is assigned a current speed and direction. Specifically, the value of speed and direction at the $n^{\text {th }}$ instance is calculated based upon the value of speed and direction at the $(n-1)^{\text {th }}$ instance and a random variable [15]. Energy consumption used for simulation is based on some numeric parameters obtained in [5].

Our prediction method is compared with the least squares minimization (LSQ) to evaluate the performance of accuracy. LSQ is a common method used for error reduction in estimation and prediction methods. LSQ solves the problem of estimating by minimizing the sum of the squares of the error terms corresponding to each distance sample. In other words, LSQ tries to get the estimate by minimizing $\sum_{i=1}^{n}\left(\left\|\hat{\phi}_{i}-p_{i}\right\|-d_{i}\right)^{2}$ where $\hat{\phi}_{n}$ is estimate and $\left\|\hat{\phi}_{i}-p_{i}\right\|$ is the Euclidean distance between the estimated coordinate of the mobile device and the beacon or receiver at position $p_{i}$ [16].

\subsection{Energy}

Fig. 7 shows the wasted energy that is defined as the amount of consumed power due to incorrect information of prediction over all nodes. Obviously energy consumption is greatly influenced by an accuracy of prediction. If the sensor nodes stay awake to track the mobile robot while the robot is moving out of the sensing range, they consume unnecessary energy. As described earlier, we can extend the network lifetime by avoiding such unnecessary energy consumption at nodes that do not need to join in tracking. This figure indicates that our scheme can decrease the number of participating nodes and thus reduce energy consumption too.

\subsection{Participating Nodes}

We first examine the number of detectable nodes in the sensing field. We can roughly compute the number of participation nodes to be involved in a mobile robot detection at a given time $t$ by 


$$
S_{t}=\frac{N \pi r_{s}^{2}}{A}
$$

where $N$ is the number of deploying nodes in whole sensor field $A$ and $r_{s}$ is the sensing range of the node. Fig. 8 shows percentage of the number of nodes that are able to detect the mobile target as varying the sensing range of the node.

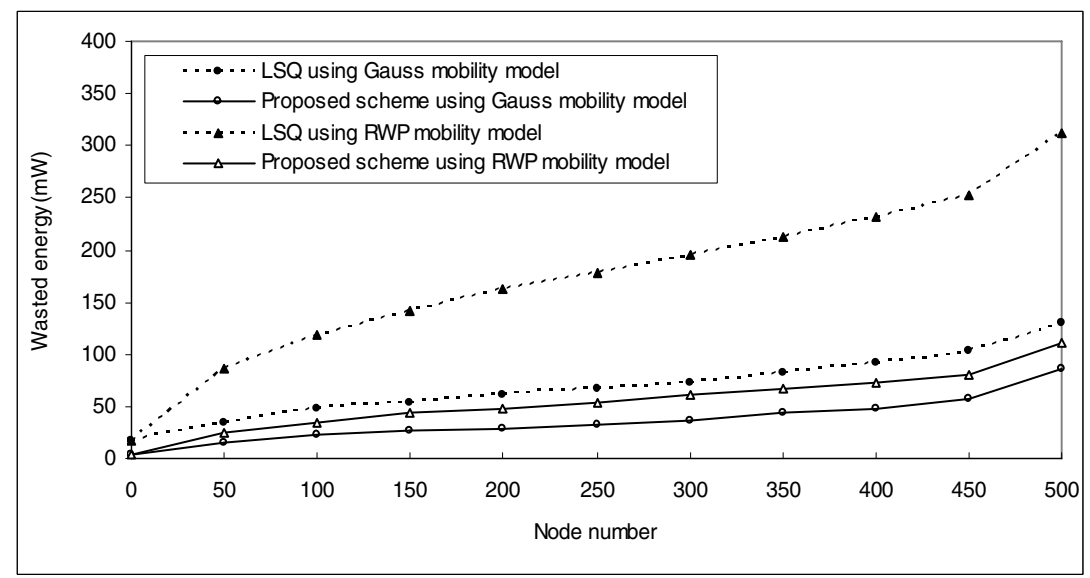

Fig. 7. Unnecessary waste of energy

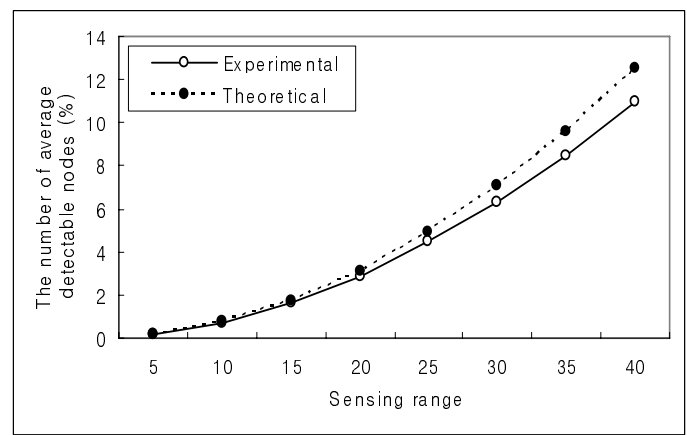

Fig. 8. Number of detectable nodes as the sensing range of node is varied

We can see that there is a sound agreement between the theoretical and experimental values. This figure also indicates we can reduce energy consumption considerably by allowing only some nodes around the mobile target to join in the tracking.

\subsection{Exposed Time}

Fig. 9 shows the percentage of the time duration when the location of the mobile robot can be known to any three or more nodes in the network. We call this exposed 
time. As previously mentioned, at least three nodes are needed simultaneously to decide the location of the target using triangulation. From this figure, we can see that the target is always detected by three sensor nodes regardless of location of the mobile target when sensing range is greater than 20 .

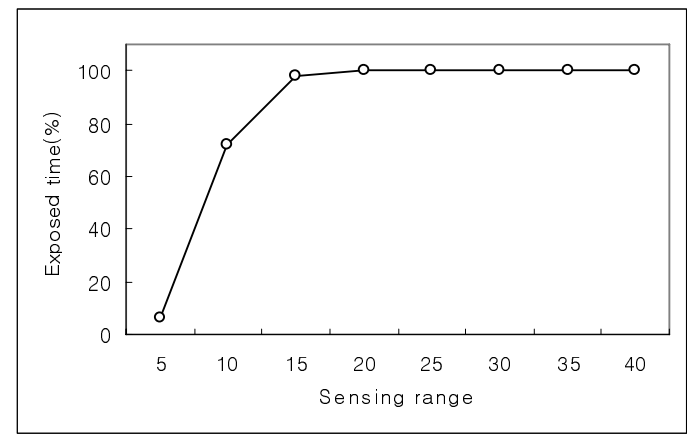

Fig. 9. Exposed time of target as the sensing range of node is varied

\section{Conclusion}

In this paper, we propose a cheap and energy efficient location tracking method of a mobile URC using a sensor network. Our method relieves the URC from the burden of complicated processing and computation to find its exact location by allowing only several nodes around the mobile URC to join the mission of location tracking. Our tracking method is based on a moving average estimator and simulations results show that our proposal can be successfully applicable to finding the location of mobile URC.

\section{References}

1. S.R. Oh, "IT Based Intelligent Service Robot," In Proceeding of the First NSF PI Workshop on Robotics and Computer Vision(RCV '03), Las Vegas, Oct. 2003.

2. H. Kim, Y.-J. Cho and S.-R. Oh, "CAMUS: A middleware supporting context-aware services for network-based robots," In Proceeding of the IEEE Workshop on Advanced Robotics and its Social Impacts(ARSO), 2005.

3. Sameer Tilak, Vinay Kolar, Nael B. Abu-Ghazaleh and Kyoun-Dong Kang, "Dynamic Localization Protocols for Mobile Sensor Networks", In Proceeding of the IEEE IWSEEASN, 2005.

4. C. Gui and P. Mohapatra, "Power conservation and quality of surveillance in target tracking sensor networks," In Proceeding of the ACM MobiCom, 2004.

5. Y. Xu, J. Winter, and W.-C. Lee, "Prediction-based strategies for energy saving in object tracking sensor networks," In Proceeding of IEEE International Conference on Mobile Data Management(MDM), 2004.

6. D. Estrin, R. Govindan, J. Heidemann, and S. Kumar, "Next century challenges: Scalable coordination in sensor networks," In Proceeding of the ACM/IEEE International Conference on Mobile Computing and Networking, 1999. 
7. T.Yan, T,He, and J, Stankovic , "Differentiated surveillance for sensor networks," In Proceeding of ACM SenSys'03, 2003.

8. T. He, S. Krishnamurthy, J. Stankovic, T. Abdelzaher, L. Luo, R. Storelu, T. Yan, L. Gu, J. Hui, and B.Krogh, "Energy-efficient surveillance system using wireless sensor networks," In Proceeding of the MobiSYS'04, 2004.

9. Y.Xu and W.-C.Lee, "On Localized Prediction for Power Efficient Object Tracking in Sensor Networks," In Proceeding of the Intionational Workshop on Mobile Distributed Computing(MDC), 2003.

10. R. Brooks and C. Griffin, "Traffic model evaluation of ad hoc target tracking algorithms," In Proceeding of the International Journal of High Performance Computer Applications, 2002.

11. R. Brooks and C. Griffin and D. S. Friedlander, "Self-organized distributed sensor networks entity tracking," In Proceeding of the International Journal of High Performance Computer Applications, 2002.

12. D. Li, K. Wong, Y.Hu and A. Sayeed, "Detection, Classification, Tracking of Targets in Micro-sensor Networks," In Proceeding of the IEEE Signal Processing Magazine, pp. 1729, March 2002.

13. S. Goel and T. Imielinski, "Prediction-based monitoring in sensor networks: tasking lessons from MPEG," In Proceeding of the ACM Computer Communication Review, 2001.

14. F. Zhao, J. Liu, J. J. Liu, L. Guibas, and J. Reich, "Collaborative signal and information processing: An information directed approach," In Proceeding of the IEEE, 2003.

15. T. Camp, J. Boleng and V. Davies, "A survey of mobility models for ad hoc network research," In Proceeding of the Wireless Communication \& Mobile Computing (WCMC): Special issue on Mobile Ad Hoc Networking, 2002.

16. A. Smith, H. Balakrishnan, M. Goraczko and N. Priyantha, "Tracking Moving Devices with the Cricket Location Systems," In Proceeding of the Mobisys, 2004.

17. H. Yang and B. Sikdar, "A Protocol for Tracking Mobile Targets using Sensor Networks," In Proceeding of the IEEE Workshop Sensor Network Protocols and Applications, (in conjunction with IEEE ICC), May, 2003.

18. HS. Kim, KJ. Han "An Energy Efficient Tracking Method in Wireless Sensor Networks", In Proceeding of the $6^{\text {th }}$ Next Generation Teletraffic and Wired/Wireless Advanced Networks(NEW2AN), May, 2006.

19. Xingbo Yu, Koushik Niyogi, Sharad Mehrotra, Nalini Venkatasubramanian, "Adaptive Target Tracking in Sensor Networks," In Proceeding of the Communication Networks and Distributed Systems Modeling and Simulation Conference (CNDS'04), 2004. 